\title{
Collisional processes and transfer of mass among the planetary satellites
}

\author{
S. Marchi ${ }^{1}$, A. Dell'Oro ${ }^{2}$, P. Paolicchi ${ }^{3}$, and C. Barbieri ${ }^{4}$ \\ 1 Dipartimento di Astronomia, Università di Padova, Vicolo dell'Osservatorio 2, 35122 Padova, Italy \\ e-mail: marchi@pd.astro.it \\ 2 Dipartimento di Fisica, Università di Pisa, piazza Torricelli 2, 56127 Pisa, Italy \\ 3 Dipartimento di Fisica, Università di Pisa, piazza Torricelli 2, 56127 Pisa, Italy \\ e-mail: paolicchi@df unipi.it \\ 4 Dipartimento di Astronomia, Università di Padova, Vicolo dell'Osservatorio 5, 35122 Padova, Italy \\ e-mail: barbieri@pd.astro.it
}

Received 12 December 2000 / Accepted 21 May 2001

\begin{abstract}
Several pairs of planetary satellites may have been involved, during the history of the Solar System, in mutual mass transfer processes. Such processes can be triggered by catastrophic collisions of a satellite (parent body) with a third object. As a consequence, the collision fragments are injected into independent orbits that can cross the trajectory of the another satellite (target). These swarms of secondary impacts may be of some importance influencing the properties of the target body. Even the formation of the atmosphere around some giant satellites may have been triggered by the gas released after the impacts of fragments onto the target's surface. Moreover, the different albedos and the different surface density of impact craters within the same satellite system may be connected to peculiar collisional phenomena, such as those we are dealing with. A quantitative modelling of the role of mass transfer processes obviously requires an estimate of how much material the parent bodies are able to supply, and under what circumstances the process may take place. A general analysis of the various pairs throughout the major satellite systems present in the Solar System has been performed in the present paper. Our analysis uses a statistical algorithm, computing, as a function of the initial properties of the fragments (masses and ejection velocities from their parent body), the mean intrinsic probability of impact, and then the mean lifetime of a fragment before impacting the target, as well as the distribution of the relative velocity. For an order-of-magnitude estimate of the available amount of mass, some simple analytical equations have been derived to evaluate the fraction of fragments from the parent body that can reach the target. These formulae allow a preliminary discrimination of the interesting cases. The pair Hyperion-Titan and the Uranus system have been analyzed in detail.
\end{abstract}

Key words. satellites - collisions - mass transfer

\section{Introduction}

Two major physical processes dominated the formation and the evolution of the Solar System, and presumably of every planetary system: gravitational interactions and collisions.

These processes are effective both on the overall evolution of the system and to determine the geological properties of individual planets and of their satellites, atmospheres and rings. In many cases some interesting properties of a body follow rather from a sort of "local" history than from the large-scale mechanisms involving

Send offprint requests to: A. Dell'Oro, e-mail: aldo@astr15pi.difi.unipi.it the whole planetary system: we cannot neglect the role of the interaction with the local environment in order to understand the intrinsic properties of a planetary body.

The cratering record of every satellite or solid planet allows to reconstruct - or, at least, to constrain - the main features of the collisional history of the body (which is often relevant information for the overall collisional evolution of the Solar System or of its subsystems). Frequent or massive cratering processes can also affect the geological properties of the body, not only in terms of the creation of observable impact craters, but also affecting the chemical composition of the surface, the rotational properties and so on. Within this context we include also the influence of collisional processes on the formation and the evolution 
of the planetary atmospheres, with natural connections to the exciting problem of the origin of organic compounds.

Two main mechanisms can cause the formation of a planetary atmosphere: accretion of the surrounding gases and degassing. The former can be invoked for the giant planets, due to their huge gravity, while the formation of the atmosphere of the terrestrial planets and of the planetary satellites is due mainly to the latter process (Brown 1952; Ozima \& Igarashi 1989). Among the 61 moons in the Solar System only five, i.e. Europa, Io, Ganymede, Titan and Triton, are known to have atmospheres. The gas release may be due to the collision of minor bodies with the surface of the considered object, involving both the gas trapped in the surface layers of the target and the gas included in the rocky projectiles. The atmospheres created by impacts may be transient: their survival depends also on the effect of erosive processes, in turn connected to the collisions (Melosh \& Vickery 1989; Walker 1986), and on the thermal escape of the gas.

The previous considerations increase the interest in analyzing the possible causes of intense collisional bombardment.

The cratering of satellite surfaces presumably has been caused both by projectiles in heliocentric orbits (as usually assumed) and also by planetocentric bodies. In fact, the morphological properties of several satellites exhibit irregular shapes (i.e. Hyperion) or peculiar surface regions (i.e. Miranda) or diffuse fractures (i.e. Ariel) (Smith et al. 1981, 1982, 1986); all these features apparently result from at least one catastrophic event in the past, with a massive fragmentation of the parent body; such events obviously inject a swarm of bodies into planetocentric orbits.

In this paper we analyze the possible transfer of mass from a satellite, ejecting fragments after a catastrophic or quasi-catastrophic collision, onto another one. We discuss the efficiency of this mechanism, its duration and the rate of mass transfer.

\section{Transfer of mass}

Within the system of satellites orbiting a central planet we choose a pair of bodies, which we will address, in the following, as parent body and target. Due to the collision with a third body (which may be an external object, such as a comet or an asteroid, moving along a heliocentric orbit, or another satellite) the parent body is destroyed or at least deeply cratered: the fragments created by such processes are injected into independent orbits around the central planet. The fragments which intercept the orbit of the target become potential projectiles for it. We wish to compute the number of projectiles able to collide with the target or, more precisely, the percentage of fragments which are potential projectiles, and how long they are expected to orbit before colliding - and thus being disrupted. We are not taking into account the possibility that the fragments formed by the disruption of a projectile may collide with more than one target (apart from the - obvious - possible reaccumulation onto the parent body).
This possibility is certainly present whenever the further dynamical evolution of fragments sensitively affects their initial keplerian orbit (see, for example, Farinella et al. 1990). A dynamical evolution of the orbits can be due to the presence of perturbing massive satellites; in particular, the close encounters can scatter the orbits. The results of our analysis are not reliable if a massive body is present between the orbits of the parent body and of the target. When the target itself is a massive body, dominating dynamically the region of interest, and the characteristic time of its perturbation is much shorter than the collisional lifetimes of the fragments, it remains - obviously able to itercept most of the fragments which pass close enough; nevertheless the estimated lifetimes of fragments may be biased (see also in the following). Moreover, a few fragments may be injected into strongly different orbits, and contribute to a moderate craterization of other satellites, not reachable on the basis of the initial conditions. In the cases we are dealing with and within the assumptions we are adopting (no further orbital evolution apart from the circulation of orbital angles) the possibility of multiple targets or massive interlopers is not too relevant, since in general only one is reachable with the adopted ejection velocity. Nevertheless we will address this point later in more detail. The orbit of a fragment depends on the ejection velocity (value and direction) from the parent body, and also on the orbital elements of the parent body at the moment of the breakup. The planetocentric orbit of the parent body can be described in terms of its Keplerian elements referred to the equatorial plane of the central planet. To compute the orbital elements of a fragment we must know all the orbital elements of the parent body at the moment of the collision, i.e. not only its semimajor axis $a_{\mathrm{PB}}$, eccentricity $e_{\mathrm{PB}}$ and inclination $I_{\mathrm{PB}}$, but also the angular elements, i.e. the longitude of the node $\Omega_{\mathrm{PB}}$, the argument of the pericenter $\omega_{\mathrm{PB}}$ and the true anomaly $f_{\mathrm{PB}}$.

Due to tidal effects and to the perturbations of the Sun and of the other bodies, the orbit is not perfectly Keplerian; nevertheless, for the sake of simplicity, we assume that the elements $a, e$ and $I$ are constant in time, while $\Omega$ and $\omega$ vary uniformly. These two conditions are the canonical hypotheses considered in most of the studies concerning the statistics of impacts among the asteroids in the Main Belt (Wetherill 1967; Bottke et al. 1994; Dell'Oro \& Paolicchi 1998). In the cases we are dealing with in the present paper, these hypotheses do not hold in general: sometimes the motion of the objects is complicated by the presence of mean-motion resonances. These resonances can either protect the objects from the collisions or can favour them. In the presence of resonances, the computation of the rate of collisions requires a numerical integration of the motion for a long time (Farinella et al. 1997), since the results obtained with a pure statistical approach, as in the present paper, are not always reliable.

The canonical hypotheses we adopt have to hold for the parent body and, consequently, for all the fragments (we 

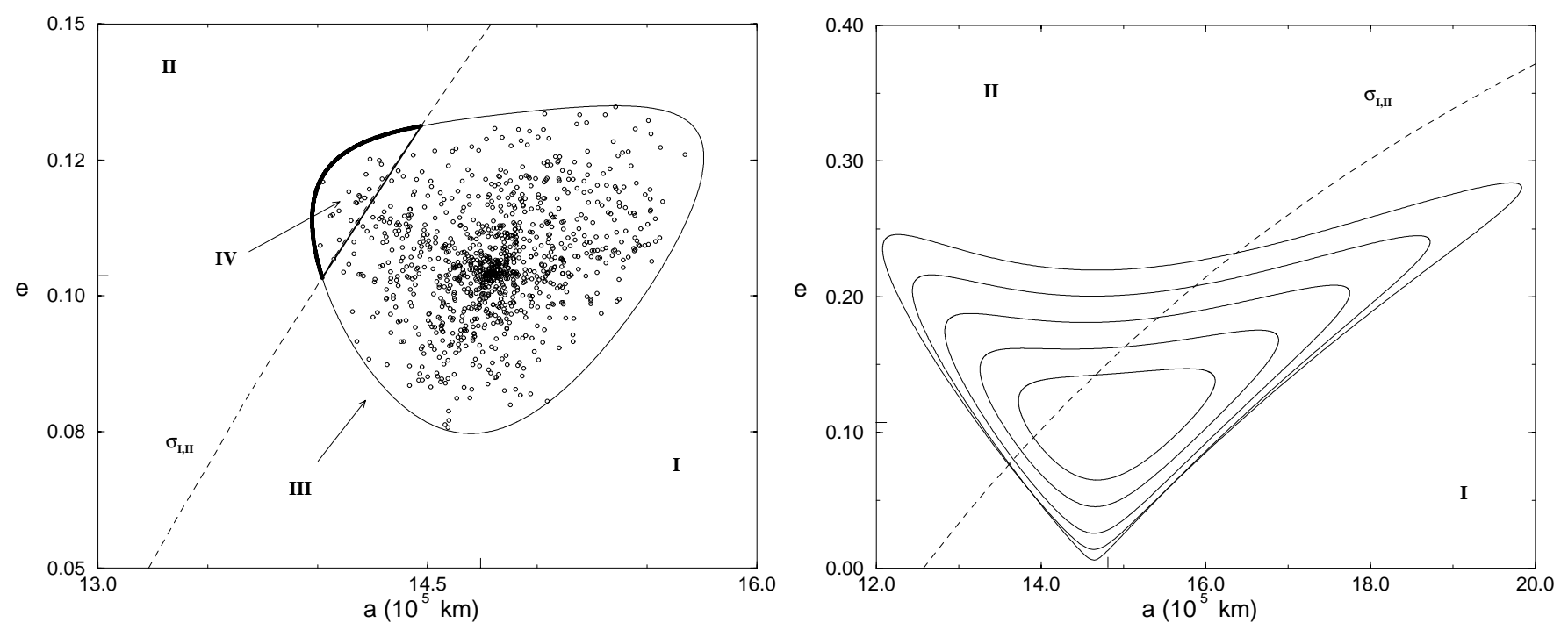

Fig. 1. Left: distribution in the plane $a-e$ of the orbits of 1000 fragment produced by an isotropic disruption of Hyperion (with $V_{\max }=0.15 \mathrm{~km} \mathrm{~s}^{-1}$ and $f_{\mathrm{PB}}=90^{\circ}$, see text for details). The regions I, II, III, and IV are defined in the text. Right: the same figure representing the lines enclosing the fragments for different values of the $V_{\max }\left(V_{\max }=0.2,0.3,0.4,0.5,0.6 \mathrm{~km} \mathrm{~s}{ }^{-1}\right.$, from the innermost to the outermost and $\left.f_{\mathrm{PB}}=90^{\circ}\right)$.

do not take into account the possibility that a fragment created from a non-resonant parent body may be captured into a resonance; a case which, nevertheless, takes place). The statistics of collisions is thus controlled only by the elements of the fragment $a, e I$, and $a_{0}, e_{0}$ and $I_{0}$ of the target. The elements $a$, e $I$ can be obtained given the ejection velocity of the fragment with respect to the parent body and the orbital elements of the parent body at the moment of breakup, including the unknown values of $f_{\mathrm{PB}}$ and of $\omega_{\mathrm{PB}}$.

\subsection{Possibility of collision}

In this section we will develop a simple analytic method for an order of magnitude estimate of the transferred mass, as a function of a few basic parameters. It will be useful for a better understanding of the problem and also for a pre-selection of the possible interesting pairs within the Solar System.

In general the possibility that a fragment can collide with the target depends only on the values of $a, e, a_{0}, e_{0}$, and the encounter is possible only if:

$\left|a-a_{0}\right| \leq a e+a_{0} e_{0}$.

In order to estimate the percentage (ratio) $\nu$ of the fragments that can collide with the target, we must introduce some assumptions on the way the parent body breaks and on the velocity distribution of the fragments.

A proper modeling of a collision between kilometersized objects is extremely difficult. Many papers, based on theoretical ideas or on the extrapolation of laboratory experiments, are present in the literature (Paolicchi et al. 1989, 1996; Melosh et al. 1992; Ryan \& Melosh 1998), for the most part devoted to the case of rocky bodies, such as asteroids. Nevertheless, up to now, many problems remain open. Most of the satellites, belonging to the systems of the giant planets, are icy and thus chemically and physically different from the Main Belt asteroids.

For these reasons, and also taking into account the purpose of the present approach, aiming only to give an order of magnitude estimate of the effect, we will make use of a strongly simplified model. We assume that the fragments are ejected isotropically, and that the distribution of the modules $V$ (the velocity attained at large distance from the parent body, when its gravity can be neglected) is uniform between the values 0 and $V_{\max }$.

As regards the isotropic model, we recall that fragments are delivered after a catastrophic collision, rather than a cratering one, which would imply a strong non isotropic model.

In principle both hypotheses could have been refined; for example it is well known that in catastrophic collisions fragments are ejected faster close to the impact point than in the antipodal region. This property has been satisfactorily modeled in the literature (see, for instance, Paolicchi et al. 1996; Tanga et al. 1999) but its introduction would require the definition of two further - a priori unknown initial conditions, say the longitude and the latitude of the impact point on the parent body surface. On the other hand, on the basis of statistical considerations, the assumption of an isotropic and uniform distribution of impact directions and impact points sounds reasonable. In other words, even if the individual breakup velocity fields are not isotropic, a sort of statistical isotropy is obtained averaging over several events, thus defining an "average event".

The isotropy assumption is sufficient for the requirements of our analysis, even if in rare cases (statistically not meaningful) the combined effect of a peculiar impact location and of an event having happened in a peculiar 

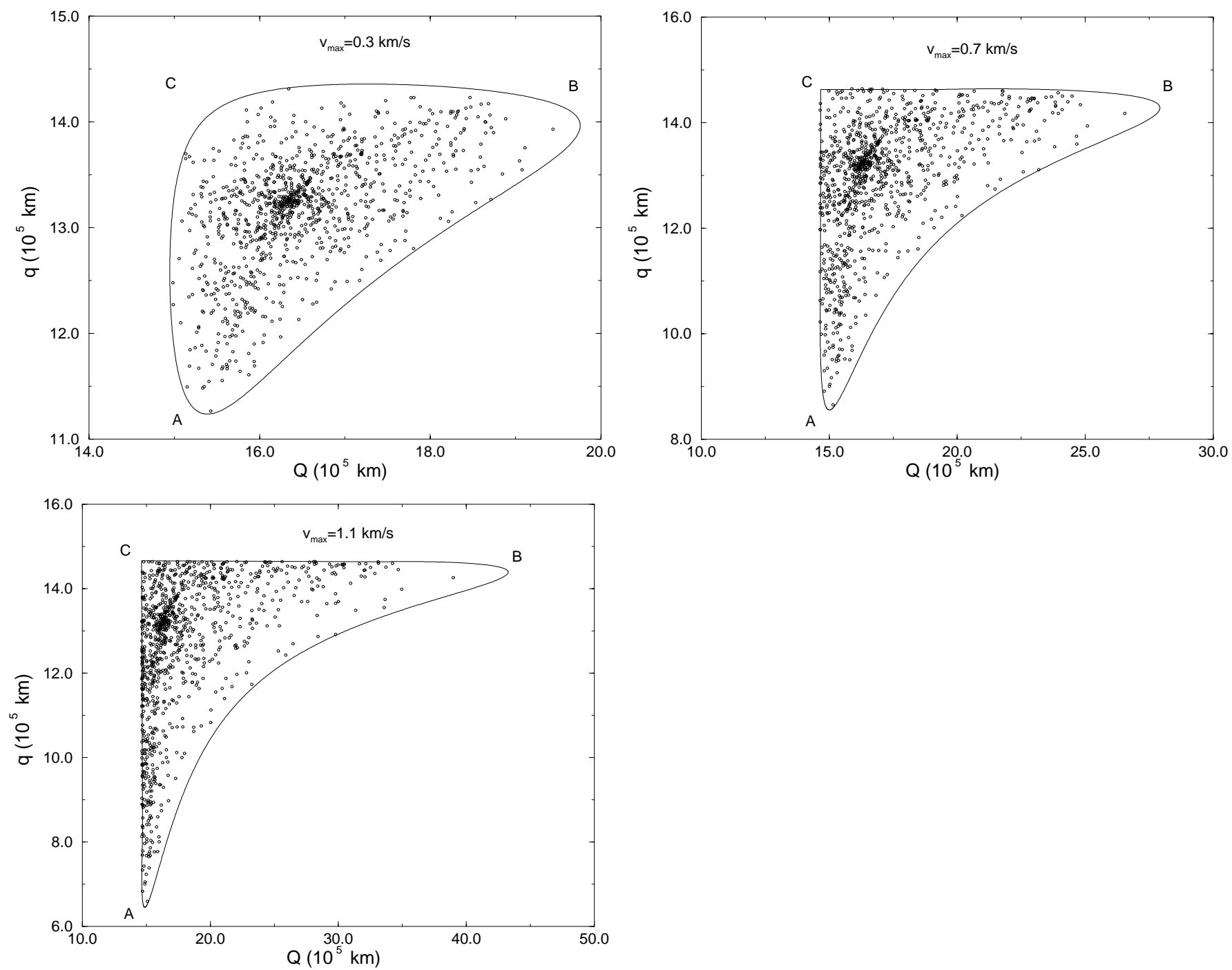

Fig. 2. Examples of distribution of the fragments in the plane $Q-q$. In each figure $f_{\mathrm{PB}}=90^{\circ}$, and the unit is $10^{5} \mathrm{~km}$.

position of a strongly eccentric orbit could also affect the estimated order of magnitude.

Concerning the uniform distribution of modules of the ejection velocity $V$, we have verified that a more realistic distribution, such as that obtained from numerical models, does not significantly affect the final results and the overall scenario; for this reason we decided to keep to the simplest approach.

Given these assumptions, the first step of our method is to evaluate analytically, for every value of $V_{\max }$, the percentage of fragments whose orbital elements fulfil Eq. (1). This analytical approach will gives us both a better characterization of orbital properties for the cluster of fragments, and a more detailed knowledge of the parameters that affected the transfer of mass process.

We decided to choose, for an introductory example of the method, the pair Hyperion (parent body) and Titan (target), since it is the only one previously discussed in the literature, and thus useful for a comparison. The orbital elements of Hyperion and Titan are respectively $a_{\mathrm{PB}}=14.81 \times 10^{5} \mathrm{~km}, e_{\mathrm{PB}}=0.1042$ and $a_{0}=12.22$ and $e_{0}=0.0292$. In Fig. 1 left, the distribution of 1000 sample fragments produced by the disruption of the satellite Hyperion, assuming $V_{\max }=0.15 \mathrm{~km} \mathrm{~s}^{-1}$ (the value 0.15 divides almost elliptical from almost triangular shapes, as shown in Fig. 1 right) is represented in the plane $a-e$ (the unit for $a$ is $10^{5} \mathrm{~km}$ ). A dashed line, labelled $\sigma_{\text {I,II }}$, separates two regions: at left the condition (1) is satisfied (region II), at right the collisions with Titan are not possible (region I). The solid line encloses the region of points corresponding to fragments whose velocity is less than $V_{\max }$ (region III). Thus the fragments that can collide with Titan belong to the intersection between the regions II and III (region IV).

The figure shows that only a small fraction of the fragments can collide with Titan. The distribution shown in the figure has been obtained assuming for the true anomaly of Hyperion a value $f_{\mathrm{PB}}=90^{\circ}$. For other values of $f_{\mathrm{PB}}$ the size of the region IV changes, and for $f_{\mathrm{PB}}=0^{\circ}, 180^{\circ}$ the region becomes a line. Obviously the size of the region depends mainly on $V_{\max }$, and, in particular, collision is possible if $V_{\max }$ is lower than a limiting 

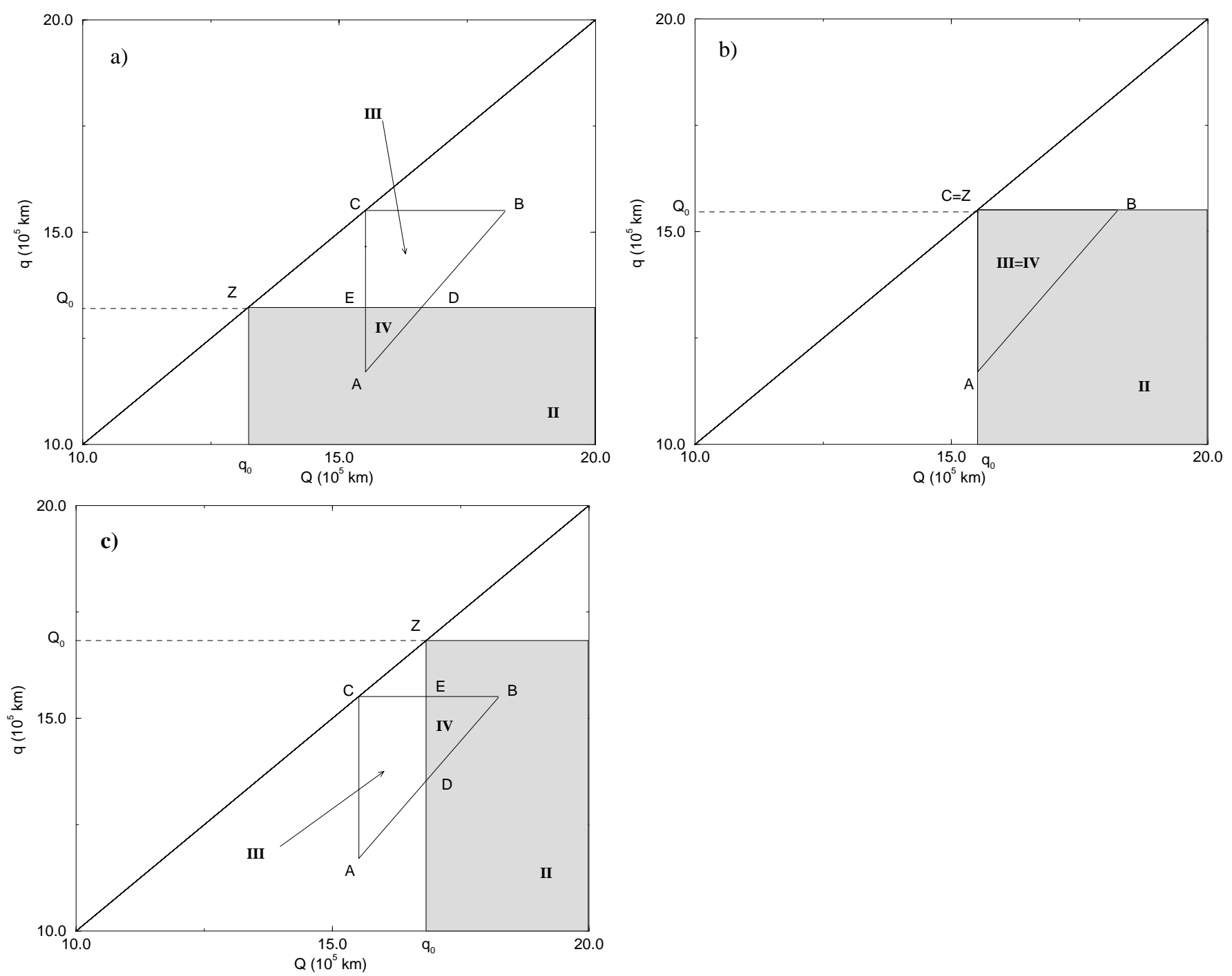

Fig. 3. Relative positions that the regions II and III can assume.

value. On the other hand, the number of fragments belonging to region IV does not depend only on the area of region IV, but also on the distribution of representative points in region III. This distribution depends on the model of breakup chosen and on the properties of the transformation from the space of the velocities to the space of the orbital elements.

For a first estimate we assume a uniform distribution within region III. In reality this hypothesis is inconsistent with the above-assumed distribution of the velocities, and we expect that the density of objects in the plane $a-e$ is maximum close to the position of the parent body, and minimum at the edge of region III. Nevertheless it is possible to take into account this systematic effect with a normalization factor (see later).

Thus the estimate of the number of potential projectiles requires essentially the estimate of the ratio:

$\nu^{\prime}=\frac{S_{\mathrm{IV}}}{S_{\mathrm{III}}}$ where $S_{\text {III }}$ and $S_{\text {IV }}$ are equal respectively to the areas of regions III and IV. The determination of $\nu^{\prime}$ in the plane $a-e$ is rather difficult because of the shape of region III (see Fig. 1) and the position of the line $\sigma_{\mathrm{I}, \mathrm{II}}$ separating regions I and II. The problem is far simpler if we work in the plane defined by the pericenter $(q)$ and the apocenter $(Q)$ distances. The quantities $q$ and $Q$ are related to $a$ and $e$ simply by:

$Q=a(1+e) \quad q=a(1-e)$.

In this way the geometric condition on the orbit intersection becomes $q<Q_{0}, Q>q_{0}$, equivalent to (1).

Also in this plane the shape of region III depends on $V_{\max }$ and $f_{\mathrm{PB}}$. Nevertheless, when $V_{\max }$ is high (the case we are concerned with) the shape can be approximated by a right triangle, with catheti parallel to the axes $Q$ and $q$ (Fig. 2). Assuming now that the distribution of fragments is uniform inside region III, when represented with the coordinates $Q-q$ (the accuracy of this assumption is essentially the same as that defined in the plane $a-e$, and the eligibility of a normalization factor is the same) we 


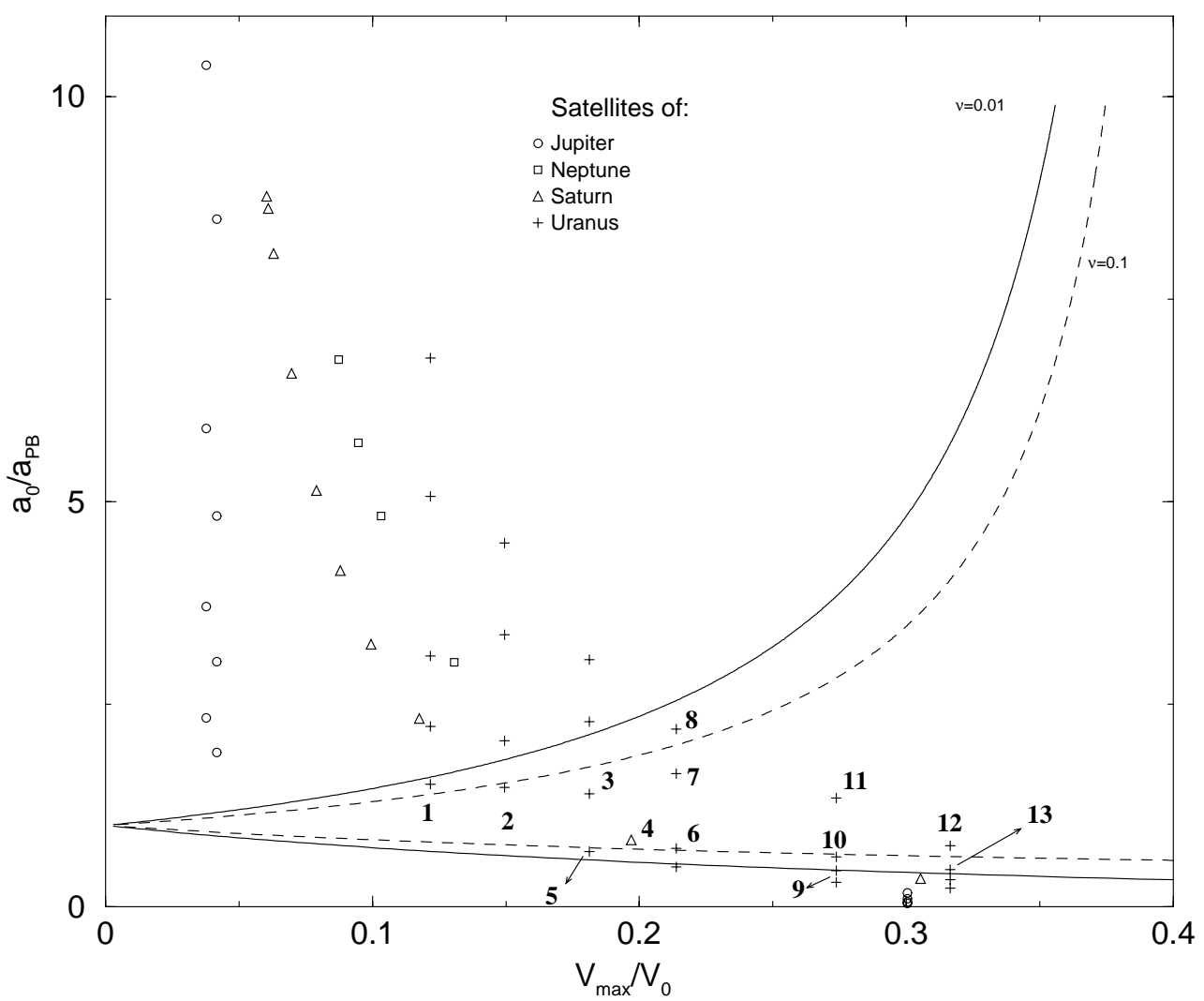

Fig. 4. Arrangement of interesting pairs in the plane $(\epsilon, \kappa)$; and curve for $\nu=$ constant, with $\nu=0.01,0.1$. The numbers correspond to the following pairs parent body-target: 1) Puck-Miranda; 2) Miranda-Ariel; 3) Ariel-Umbriel; 4) HyperionTitan; 5) Ariel-Miranda; 6) Umbriel-Ariel; 7) Umbriel-Titania; 8) Imbriel-Oberon; 9) Ariel-Titania; 10) Titania-Umbriel; 11) Titania-Oberon; 12) Oberon-Titania; 13) Oberon-Umbriel; 14) Phoebe-Japetus (not included in the figure because of the high value of $\left.V_{\max } / V_{0}\right)$.

have only to recompute the ratio $\nu^{\prime}$, and to derive, from this value, the estimated fraction of potential projectiles $\nu$.

The computation above can be performed for a generic orbit of the parent body; nevertheless, in order to obtain the simplest possible criterion, we discuss the case of circular orbits (i.e., $e_{\mathrm{PB}}=e_{0}=0$; it entails also $Q_{0}=q_{0}=a_{0}$ ); we will discuss in the Appendix how the general approach introduces usually moderate differences.

The first step is to define the position and to compute the area of the triangle (see Fig. 3). The vertices of the triangle are determined in the following way. The right angle (vertex $\mathrm{C}$ ) is on the line $q=Q$. It corresponds to the fragment with a circular orbit. Its orbit must intercept the point of the breakup and then the value of its semimajor axis must be $Q_{\mathrm{C}}=q_{\mathrm{C}}=a_{\mathrm{PB}}$.

Moreover, for the same reason, the other fragments must have the apocenter $Q>Q_{\mathrm{C}}$ and the pericenter $q<$ $q_{\mathrm{C}}$; thus the catheti of the triangle lie on the lines $Q=$ $Q_{\mathrm{C}}$ and $q=Q_{\mathrm{C}}$. The vertices $\mathrm{A}$ and $\mathrm{B}$ depend on the maximum and minimum energy that the fragments can have. In fact, the energy $\mathcal{E}$ being equal to $-\mu /(Q+q)$ where $\mu=G M_{\odot}$, the lines of constant energy are:

$q=-Q-\frac{\mu}{\mathcal{E}}$

In this way, the fragment with minimum $\mathcal{E}$ corresponds to the point $\mathrm{A}$, while the one with maximum $\mathcal{E}$ corresponds to B. It is easy to show that the minimum and maximum energy for the fragments with the distribution of velocity defined above are:

$\mathcal{E}_{\mathrm{MIN}}=\frac{\mu}{2 a_{\mathrm{PB}}}\left(-1+\epsilon^{2}-2 \epsilon\right)$

$\mathcal{E}_{\mathrm{MAX}}=\frac{\mu}{2 a_{\mathrm{PB}}}\left(-1+\epsilon^{2}+2 \epsilon\right)$

where $\epsilon=V_{\max } / V_{0}$, and $V_{0}$ is the velocity of the parent body at the moment of the breakup (note that $\epsilon>0$ ). Eqs. (4)-(5) with Eq. (3) give:

$Q_{\mathrm{A}}=a_{\mathrm{PB}} \quad q_{\mathrm{A}}=\frac{\mu}{\mathcal{E}_{\mathrm{MIN}}}-a_{\mathrm{PB}}$

$Q_{\mathrm{B}}=-\frac{\mu}{\mathcal{E}_{\mathrm{MAX}}}-a_{\mathrm{PB}} \quad q_{\mathrm{B}}=a_{\mathrm{PB}}$

and the area of the triangle, i.e. of region III, is:

$$
\begin{aligned}
S_{\mathrm{III}} & =\frac{1}{2}\left(q_{\mathrm{C}}-q_{\mathrm{A}}\right)\left(Q_{\mathrm{B}}-Q_{\mathrm{C}}\right) \\
& =-\frac{1}{2}\left(2 a_{\mathrm{PB}}+=\frac{\mu}{\mathcal{E}_{\mathrm{MIN}}}\right)\left(2 a_{\mathrm{PB}}+\frac{\mu}{\mathcal{E}_{\mathrm{MAX}}}\right) .
\end{aligned}
$$

The last step is to determine the area of region IV, that is the intersection between region II and region III. In Fig. 3 an example of partition of the plane $Q-q$ in the five regions 
is represented. According to condition (1) the region II is determined by the point $Z$ of coordinates $\left(a_{0}, a_{0}\right)$, lying on the bisector. Having fixed the position of the triangle, depending on the elements of the parent body and $V_{\max }$, the area of region IV can be determined knowing the semimajor axis of the target $a_{0}$. As shown by Fig. 3 , three interesting cases can take place. The case a) holds when $\mathrm{A} \in \mathrm{II}$ and $\mathrm{B} \notin \mathrm{II}$, the case $\mathrm{b}$ ) when $\mathrm{A} \in \mathrm{II}$ and $\mathrm{B} \in \mathrm{II}$, and the case c) when $\mathrm{A} \notin \mathrm{II}$ and $\mathrm{B} \in \mathrm{II}$. In case b), being $S_{\mathrm{III}}=S_{\mathrm{IV}}$, the parameter $\nu$ is trivially equal to 1 . The other cases can be easily treated studying the intersection DE along the line $q=a_{0}$ (case a) and computing the area of the triangle $\mathrm{ADE}$, or the line $Q=a_{0}$ (case c) and the area of the triangle $\mathrm{BDE}$, and obtaining

$\nu^{\prime}=\left(\frac{\kappa+\frac{2}{-1+\epsilon^{2}-2 \epsilon}+1}{\frac{2}{-1+\epsilon^{2}-2 \epsilon}+2}\right)^{2}$

for case a, and

$\nu^{\prime}=\left(\frac{\kappa+\frac{2}{-1+\epsilon^{2}+2 \epsilon}+1}{\frac{2}{-1+\epsilon^{2}+2 \epsilon}+2}\right)^{2}$

for case c, where $\kappa=a_{0} / a_{\mathrm{PB}}$.

The above estimate of the area ratio (based on the assumed triangular shape) is approximate; moreover to assume that this value equals the fraction of useful projectiles (i.e. $\nu^{\prime}=\nu$ ) is optimistic, due to the strong nonuniformity of the distribution of the objects in region III. We analyzed in detail the case of the pair Titan-Hyperion, employing the full numerical approach to estimate $\nu$. In that case, we found that $\nu \sim 0.5 \nu^{\prime}$. Thus we have decided, for our limited-purpose first estimate, to assume that in general $\nu / \nu^{\prime} \sim 0.5$ (theoretical arguments show, as previously discussed, that this factor must be in general smaller than one). Apart from the uncertainties on the normalization factor, Eqs. (9) and (10) are a very efficient tool to compute the transfer rate: they are analytically simple and depend only on a couple of adimensional parameters, i.e. $\kappa$ and $\epsilon$.

In the Solar System, several satellite systems may be influenced by mass transfer processes. On the other hand, not all the possible pairs of objects have the right configuration, and a preliminary selection must be done. We have considered as parent bodies only the objects with a radius greater than $50 \mathrm{~km}$, and as targets bodies with a radius greater than $300-400 \mathrm{~km}$. In this way about fifty target-parent body pairs can be identified. In Fig. 4 these pairs have been represented in the plane $(\epsilon, \kappa)$ under the assumption $V_{\max } \sim 1 \mathrm{~km} \mathrm{~s}^{-1}$. This velocity value is large enough to take into account the existing uncertainties on the physical parameters of the impact processes: we can trust that, whenever a relevant mass of fragments, coming from the disruption of a body in the hundred kilometer size class, escapes much faster than $1 \mathrm{~km} \mathrm{~s}^{-1}$, no detectable parent body remnant is preserved. Later in the paper, for the purposes of completeness, we will relax this limit value to $1.5 \mathrm{~km} \mathrm{~s}^{-1}$ in a few cases. In the figure the curves $\nu=0.1$ and $\nu=0.01$ have also been represented. With this analysis one can conclude that in the Jupiter and Neptune systems we have no interesting pairs, while relevant cases are present in the Saturn system (the well known Titan-Hyperion case but also PhoebeJapetus) and in the Uranus system. Thus the Titan atmosphere is the only one which may have been created by the collisional processes discussed in the present paper.

Nevertheless, we stress that the mass transfer processes may take place according to different rules; thus, pairs which, according to our analysis, cannot be linked together (or only under the assumption of unrealistic ejection velocities) might be linked with more complex dynamical trails, involving resonances and close encounters with very massive satellites. In some rare cases, one might even obtain meaningful transfer percentages for additional pairs, as suggested by some authors (see Zahnle et al. 1999, 2000). This kind of analysis cannot be included in the present, simplified model, providing a significant but potentially incomplete list of the interesting cases.

Note also that the values have been computed assuming that all the fragments which are geometrically allowed will impact the target, neglecting the possibility that the fragment also may recollide with the parent body or other possible targets (say, other satellites). This assumption is wrong when the parent body or another satellite of the system, reachable with the ejection velocities we are assuming, is far larger than the nominal target. Nevertheless, very massive parent bodies have been excluded due to the high velocity required to overcome their gravitation, and very massive "interlopers" are not present in our cases. In the other cases the simplification above may lead to an overestimate of, say, a factor 2 or less of the amount of eligible mass, not affecting our mainly qualitative conclusions.

\subsection{Lifetimes of the fragments}

The quantity $\nu$ does not give information about the rate of collisions with the target, i.e. the estimated number of fragments, among those fulfilling the condition (1), that collide with the target in the time unit. The knowledge of the dynamical behaviour of the object is required for this goal. We assume that the elements $a, e$ and $I$ are fixed, while $\Omega$ and $\omega$ circulate uniformly, both for the projectile (fragments) and for the target. As we have discussed before, this assumption is not always acceptable, and its validity has to be ascertained for the cases under scrutiny.

There are some different methods to compute the probability of collision. The simplest is known as the "particle in a box" model. More accurate methods are by Wetherill (1967), and Greenberg (1982), this latter representing the standard one in the field of statistical studies. More recent approaches are of Vedder $(1996,1998)$ and Dell'Oro \& Paolicchi (1998). We do not discuss here the differences among these methods which, for the aims of this work and given the dynamical assumptions listed above, are 
essentially equivalent. Here we adopt the method by Dell'Oro \& Paolicchi (1998).

We refer to the paper above for the details of the method. Here we recall only that the main output of the statistical analysis is the so-called "intrinsic probability of impact" $P_{\text {i }}$, i.e. the number of impacts between two objects in the unit time if the sum of their radii is assumed equal to $1 \mathrm{~km}$. $P_{\mathrm{i}}$ is usually computed in $\mathrm{km}^{-2}$ and $\mathrm{yr}^{-1}$. In order to obtain the real number of collisions in unit time, it is sufficient to multiply $P_{\mathrm{i}}$ by the squared sum of the radii. On the other hand if the target is much larger than the projectile, the radius of this latter can be ignored and the rate of impact $P$ is equal to $P_{\mathrm{i}} R_{0}^{2}$, where $R_{0}$ is the radius of the target.

The mean lifetime of the projectile $T$ is simply equal to $1 / P$. In Dell'Oro \& Paolicchi $(1998)$ the rate $P$ is computed as the integral of a differential quantity $p$ depending on the orbital elements $\Omega_{0}, \omega_{0}$ and $f_{0}$ of the target (the elements $a, e, I$ and $a_{0}, e_{0}, I_{0}$ are supposed fixed)

$P=\pi R_{0}^{2} \int p\left(\Omega_{0}, \omega_{0}, f_{0}\right) \mathrm{d} \Omega_{0} \mathrm{~d} \omega_{0} \mathrm{~d} f_{0}$.

Every set of values of the target elements identifies a particular collision (sometimes no collision occurs), for which the elements of the projectile $\Omega, \omega$ and $f$, as well as the relative velocity $v_{\text {rel }}$ at the moment of the encounter (not taking into account the acceleration due to the mutual gravitational interaction) are automatically determined. In the computation of $P$ only the geometrical cross-section of impact, $\left(\pi R_{0}^{2}\right)$ is usually considered; but if the target is very large its gravitational attraction cannot be neglected, and we must take into account the contribution of the "gravitational focusing": the cross-section is corrected, replacing the geometrical radius $R_{0}$ with the "gravitational radius" of the target $\mathcal{R}_{0}$, equal to

$\mathcal{R}_{0}=R_{0} \sqrt{1+\frac{v_{\mathrm{e}}^{2}}{v_{\text {rel }}^{2}}}$

where $v_{\mathrm{e}}^{2}$ is the escape velocity of the target. Since the relative velocity depends on the particular collision under consideration, the integrand function in (11) has to be weighed with the gravitational radius, so that the probability of collision $\mathcal{P}$ is given by

$\mathcal{P}=\pi R_{0}^{2} \int p\left(\Omega_{0}, \omega_{0}, f_{0}\right)\left(1+\frac{v_{\mathrm{e}}^{2}}{v_{\text {rel }}^{2}}\right) \mathrm{d} \Omega_{0} \mathrm{~d} \omega_{0} \mathrm{~d} f_{0}$

(here also $v_{\text {rel }}$ depends on $\Omega_{0}, \omega_{0}$ and $f_{0}$ ). In this way the mean lifetime computed using the geometrical crosssection is substituted by the gravitational mean lifetime $\mathcal{T}$, equal to $1 / \mathcal{P}$. The result given by (13) must be taken with caution. The effect of the gravitational focusing causes an increase of the effective radius of the target regarding the impacts. Nevertheless in a close approach with a massive object the gravity has the effect not only of favouring the collision but also, if the collision does not occur, of perturbing the original orbit of the projectile. The adjustments of the projectile orbits induced by the target modify the probability of impact in a way that is not taken into account in our simple model of the gravitational influence.

A qualitative argument may be introduced. The alteration of the dynamical properties of a population of small bodies orbiting around a central mass under the effect of another large mass orbiting in the same region has been widely discussed within the studies devoted to the formation of the Solar System (see, in particular, Safronov $1972,1979)$. It has been shown that the system dynamically evolves toward a steady state; the mean relative squared velocity - at infinity - of encounters converges towards a fraction $\theta$ (usually not much smaller than unity) of the escape velocity of this body. Thus, when the steady state has been reached, the correction of the cross section due to gravitational focusing is a factor $1+\theta$ which cannot exceed unity but by a moderate amount. According to this argument, whenever the initial relative velocity is very small (in units of the escape velocity of the target), we can expect an early burst of collisions (according to the full gravitational focusing correction) plus a following collisional rate moderately exceeding that one computed without the gravitational focusing correction. Note also that, if the escape velocity of the target is very large, the evolution subsequent to a few close encounters may lead the fragments into very different orbits, eventually intersecting other bodies of the systems, which were unreachable under the original conditions.

From the relative velocity $v_{\text {rel }}$, provided by the statistical analysis, the real impact velocity $v_{\mathrm{i}}$ on the surface of the target can be evaluated throughout the known relationship

$v_{\mathrm{i}}^{2}=v_{\mathrm{rel}}^{2}+v_{\mathrm{e}}^{2}$

and the distribution of the $v_{\text {rel }}$ can be corrected according to Eq. (14) in order to obtain the distribution of $v_{\mathrm{i}}$. In the same way $V_{\max }$ should be decreased by the amount lost in escaping from the parent body. So we consider $V_{\max }$ the velocity at infinity that can be obtained by the breakup velocity $V_{\max }^{\prime}$ immediately after the fragmentation by

$V_{\max }^{2}=V_{\max }^{\prime 2}-v_{\mathrm{e}, \mathrm{PB}}^{2}$

where $v_{\mathrm{e}, \mathrm{PB}}^{2}$ is the escape velocity of the parent body; obviously $V_{\max }^{\prime 2}>v_{\mathrm{e}, \mathrm{PB}}^{2}$, otherwise no transfer of mass is possible and a total reaccumulation occurs.

All the statistical quantities defined here refer to a given pair target-projectile, and so they are evaluated only for a particular fragment placed in region IV. On the other hand the probabilities $P$ and $\mathcal{P}$, and so the distributions of the relative velocity, depend on $a, e, I$. The information that we are interested in is connected to the total effect on the target produced by all the available impactors, i.e. all the fragments in region IV. In principle, the total rate of collisions suffered by the target $P_{\text {TOT }}$ (or $\mathcal{P}_{\text {TOT }}$, if we consider the gravitational focusing) is given by the sum of $P$ (or $\mathcal{P}$ ) for every projectile, and the same holds for the total distribution of the velocities of impact on the target. On the other hand, given the uncertainties in our modelling, 


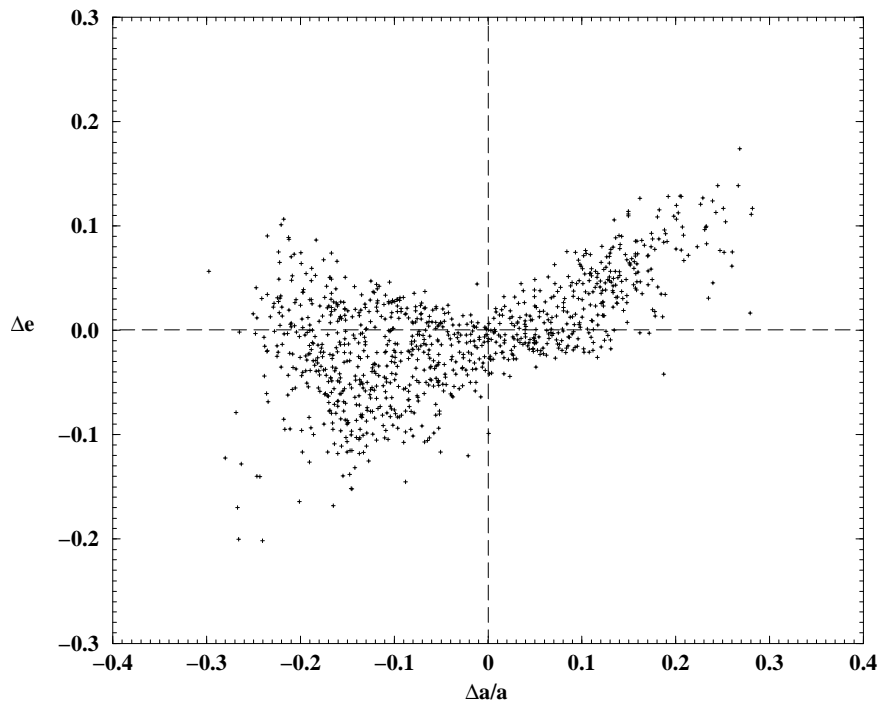

Fig. 5. Scattering diagram of Hyperion's fragments crossing the orbit of Titan and caused by the gravity of Titan. This diagram has been constructed computing the distribution of the mean variations of the elements $\Delta a$ and $\Delta e$, following a close encounter with Titan, suffered by the fragments of the Fig. 1.

the most meaningful outcome is a mean rate of collision obtained sampling region IV with a uniform distribution of projectiles. The interesting results are then the mean rates $\langle P\rangle$ and $\langle\mathcal{P}\rangle$, and from them the sample mean lifetimes $\langle T\rangle$, equal to $1 /\langle P\rangle$, and $\langle\mathcal{T}\rangle$, equal to $1 /\langle\mathcal{P}\rangle$. It is possible to introduce these averages because the probability and velocity of impact of a given projectile are not correlated with its mass, so that we can treat separately the distribution of the masses and the distribution of the velocity during the impact phase with the target.

\subsection{Suitability of the statistical approach}

Two questionable approximations that we have employed may affect the reliability of our results. The first approximation is to assume values of $a, e, I$ constant in time and uniform distribution of the angles $\omega$ and $\Omega$. The second consists of not taking into account the effect of gravitational focusing and scattering involving the targetprojectiles and parent body-projectiles pairs. In principle, within the formalism of Dell'Oro \& Paolicchi (1998), the assumption of uniformity of the angles $\omega$ and $\Omega$ can be relaxed, and the effect of gravitational scattering can be taken into account. In this subsection we intend to discuss synthetically these two problems, reporting a few preliminary computations showing that our basic results remain essentially valid. A more profound analysis will be done in a forthcoming paper, devoted to similar effects affecting the collisions involving asteroids (Dell'Oro et al. 2001, in preparation).

In general the orbital elements $a, e$ and $I$ are not constant in time, but oscillate around mean values: from a statistical point of view using these mean values does not af- fect severely the estimated probabilities of collision, apart from a few particular cases. In the presence of dynamical resonances some orbits may be highly unstable, but the fraction of unstable objects within the swarm of projectiles is generally small. The problems concerning the distribution of the angular elements $\Omega$ and $\omega$ are slightly more troubling. There are reasons to believe that these elements may not be uniformly distributed. The gravitational influence of an external body can alter the distribution of the orbital orientations, causing a clustering of the lines of apsides around the line of apsides of the perturbing body. As an example we can recall the distribution of the orbits of the Main Belt asteroids noted by Kresák (1967). Note that, in the absence of other perturbing bodies, the circulation of orbits may be caused also by the oblateness of the central planets (see for example Bertotti \& Farinella 1990). The uniformity is attained after an interval of time from the projectile formation (when the angular elements are peaked around the elements of the parent body) depending on the mean difference of the circulation rates. If the lifetime of the projectiles exceeds this "randomization" time we can fully rely on the uniformity assumption; otherwise, we have to relax it. According to Gladman et al. (1995), this latter case is expected to be frequent for the swarms of fragments in the satellitary systems.

On the other hand, the non-uniformity of the angles affects the results only when the collisions among bodies belonging to the same swarm are considered. If the target is external to the projectile swarm and its dynamical history is not correlated with that of the projectiles, any nonuniformity of the swarm's angular elements (i.e. whenever the elements of the projectiles are correlated each other) does not affect the probability of collision (see Dell'Oro et al. 2001 for a discussion). Thus the problem concerns essentially the re-impacts of the projectiles with their parent body, dynamically belonging to the swarm. A statistical evaluation of the probability can be performed with the statistical algorithm explained in Dell'Oro \& Paolicchi (1998); the problem will be dealt in detail in a forthcoming paper (Dell'Oro et al. 2001, in preparation). We are presently anticipating a few basic ideas, for the use of some tests, whose results we will discuss. The dynamical behaviour of the angles $\omega$ and $\Omega$, at any epoch between the formation of the fragments' swarm and the epoch of the randomization of their orbits can be approximately represented by a distribution of probability $\psi(\omega, \Omega)$ peaking around the original values of the angles and whose width depends on the time since the break-up. A short time is required for the randomization of the mean anomalies of the swarm. After this time, the probability of collision can be estimated within the model and is larger with respect to the steady-state value; nevertheless, the period in which the value is far larger remains rather short; thus the results are not qualitatively different, with the only exception being cases in which the parent body is far larger than any potential target. These cases are of no relevance for the present work. 

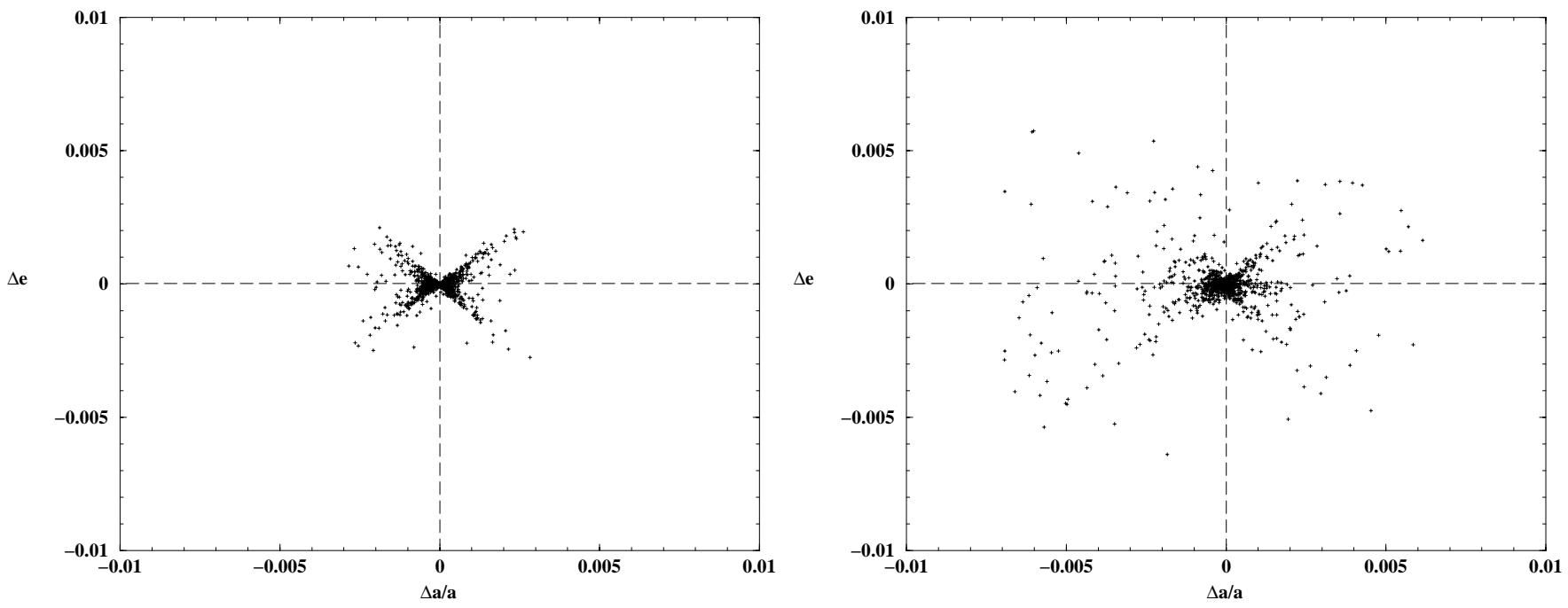

Fig. 6. Scattering diagram of Hyperion's fragments caused by the gravity of Hyperion. This diagram has been constructed with the same method used for Fig. 5 but using Hyperion as the scattering center in place of Titan. In the left plot the statistical distribution of the angles $\omega$ and $\Omega$ is supposed uniform, while in the right plot we have take into account a peaked distribution of those angles.

The problem is more complex and interesting when we combine the above discussed effect (the increased collisional probabilities with the parent body) with the latter one (scattering). The fragments are not only colliding but are also scattered by the various potential targets; in general these encounters alter the motion of the projectiles, altering the subsequent evolution of the swarm. This effect is relevant in general, but might be dramatically enhanced during the transient phase. It should be argued that the repeated scatterings with the parent body may affect the distribution of the orbits of the ejecta, through focusing processes, or by rapidly increasing their relative velocities with respect to the original orbit of the parent body. In this latter case, the effective ejection speed to be used in our computations should be the final one resulting from this transient stage; it might exceed our limit of $1 \mathrm{~km} \mathrm{~s}^{-1}$.

In general, the scattering effects may be extremely important when the scatterer is a large body. For example, we can consider the case of transfer of mass from Hyperion to Titan. We have studied the scattering produced by Titan on the orbits of the fragments coming from Hyperion. In Fig. 5 the distribution of the variations of the elements $\Delta a / a$ and $\Delta e$ for 1000 close encounters with Titan is represented. We define "close encounter" as an approach within a distance smaller than the Hill's radius of the scattering body. The fragments have been ejected by Hyperion isotropically with $V_{\max }=1 \mathrm{~km} \mathrm{~s}^{-1}$. The orbits of the fragments are strongly affected. A word of caution is thus required to assess in general our lifetime estimates, while the fraction of impacting projectiles should not change very much.

In Fig. 6 left, we show the same type of scattering diagram as Fig. 5 for the scattering produced by Hyperion on its fragments. The effect is two orders of magnitude smaller; in addition, we note a peculiar cross-shaped fea- ture. This feature can be easily explained on the basis of the distribution of the direction of the encounter velocity. Introducing the distribution $\psi(\omega, \Omega)$ the situation changes. The more peaked is $\psi$, the more similar to the primordial ones are the orbits of the fragments. This means that the distribution of the encounter velocity is isotropic and its magnitude decreases. In this condition the crossshape in the scattering diagram disappears and the magnitude of the scattering increases. Figure 6, right, shows the scattering diagram when the amplitude of $\psi$ is around $1 \mathrm{deg}$. The distributions in Figs. 5 and 6 are not a representation of the result of an evolutionary process but rather of the characteristics and magnitude of the scattering at a particular epoch. Following the same approach as Greenberg (1982) we have estimated that the standard deviation of the scattering produced on Hyperion's fragments by the gravity of Hyperion during their entire lifetime is of the order of $10^{5} \mathrm{~km}$ in the semimajor axis and $5 \times 10^{-2}$ in eccentricity, i.e. about ten per cent of the original dimension of the fragments' cloud in the plane $a-e$ (see Fig. 1), with the number of close encounters per object around 1. This is equivalent to an increase in $V_{\max }$ of the same percentage, largely acceptable for the precision in the evaluation of mass transfer we performed in this work. Moreover, as can be seen in Fig. 6, the scattering can either increase or decrease the semimajor axis and eccentricity without any systematic perturbation of the orbits, nor focusing effects.

Thus, within the limitations discussed in this subsection and elsewhere in the paper, our - approximate estimates and our qualitative conclusions should not be severely affected.

\section{Results and conclusions}

The qualitative analysis performed in the previous section is the starting point for a detailed analysis of the 


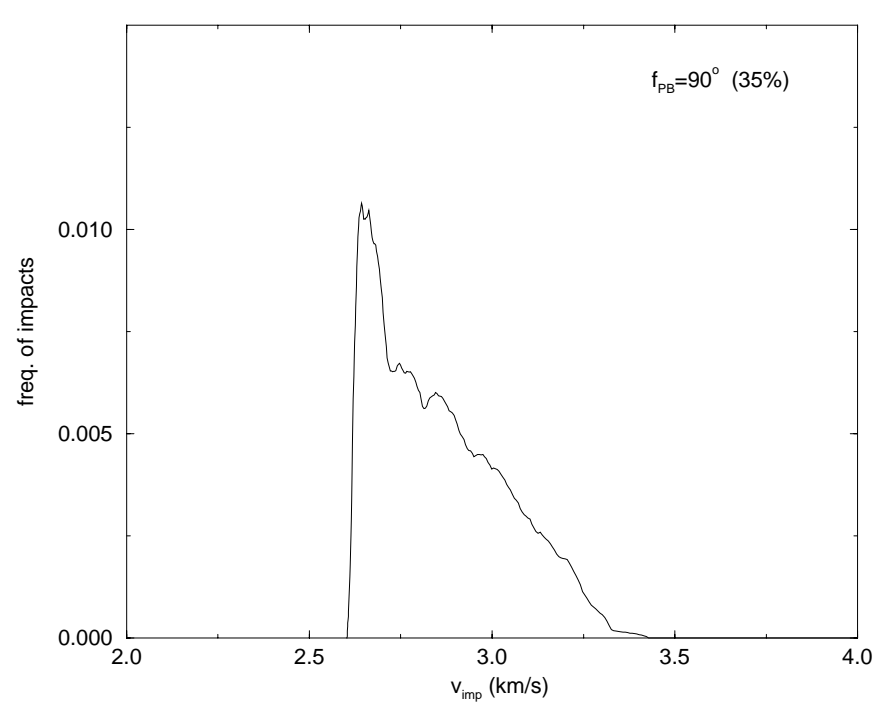

Fig. 7. Distribution of the impact velocity between Titan and a sample of projectiles. The distribution of the velocities of the projectiles is isotropic with respect to the parent body (Hyperion), $V_{\max }=1 \mathrm{~km} \mathrm{~s}^{-1}$ and the anomaly of Hyperion at the moment of the breakup is $f_{\mathrm{PB}}=90^{\circ}$. The percentage of fragments that can collide with Titan is about $35 \%$ (see Table 2).

interesting pairs. We extend our analysis to a few additional cases, for which the limit of $1 \mathrm{~km} \mathrm{~s}^{-1}$ for $V_{\max }$ is not fulfilled. Firstly we compute, for every pair, the values of $V_{\max }$ for which $\nu=0.1$ and $\nu=0.01$, as can be easily done inverting the final formulas (9) and (10), and introducing the normalization factor (0.5) defined above. For all the considered cases, the formulae for circular orbits were used, but for the cases with high eccentricity the computation has been repeated using the corrected formulae (see appendix). In Tables 1 and 3 we give the values in $\mathrm{km} \mathrm{s}^{-1}$ that $V_{\max }$ must have for a given $\nu$. The cases for which $e_{\mathrm{PB}}>0.05$ are shown in the Tables with a*. The symbol $<$ in place of the numbers warns us that the formulae do not apply: for the given $\nu$ a negative value of $q_{\mathrm{A}}$ should be required. Note that the equations may have solutions for different values of $\nu$ : for example in the cases of the pairs Phoebe-Titan and Phoebe-Hyperion (Table 1) a value of $\nu=0.001$ is provided by velocities of the order of $1 \mathrm{~km} \mathrm{~s}^{-1}$. Nevertheless we did not take into account such possibilities: our main interest is the possibility of massive mass transfers.

In Tables 2 and 4 the average lifetimes (in years) of projectiles vs. impact on the target in some of the most interesting systems are listed. The lifetimes are computed assuming a isotropic breakup with a particular $V_{\max }$ and for some values of $f_{\mathrm{PB}}$ (in the case of eccentric $\mathrm{PBs}$ ), and the average takes into account, obviously, only the bodies lying in region IV. The number of virtual fragments is 1000 in our simulations; in brackets we give $N$, the number of objects in region IV. The ratio $N / 1000$ should be compared to the analytical value $\nu$.
Table 1. Velocity $V_{\max }\left(\mathrm{km} \mathrm{s}^{-1}\right)$ among interesting pairs of Saturn, with circular orbit formulae (see text). The values in roman have been computed assuming $\nu=0.01$, while the values in italic are for $\nu=0.1$. Moreover the high eccentricity case is shown: the three values correspond to $f_{\mathrm{PB}}=0^{\circ}, 90^{\circ}, 180^{\circ}$.

\begin{tabular}{c||ccc}
\hline \hline \multirow{2}{*}{ Target } & \multicolumn{3}{c}{ Parent body } \\
Hyperion* & Iapetus & Phoebe* $^{*}$ \\
\hline Titan & 0.26 & 1.21 & $1.18^{+}$ \\
& 0.42 & $<$ & $<$ \\
\hline Hyperion & & & $1.06^{+}$ \\
& & $<$ \\
\hline Iapetus & & & $<.81$ \\
& & & $<$ \\
\hline \hline
\end{tabular}

high eccentricities

\begin{tabular}{|c|c|c|c|c|c|c|}
\hline \multirow{4}{*}{$\frac{\text { Target }}{\text { Titan }}$} & \multicolumn{6}{|c|}{ Parent body } \\
\hline & \multicolumn{3}{|c|}{ Hyperion } & \multicolumn{3}{|c|}{ Phoebe } \\
\hline & 0.36 & 0.26 & 0.11 & & & \\
\hline & 0.48 & 0.38 & 0.26 & & & \\
\hline \multirow[t]{2}{*}{ Hyperion } & & & & $1.25^{+}$ & $1.04^{+}$ & $0.84^{+}$ \\
\hline & & & & $<$ & $<$ & $<$ \\
\hline \multirow[t]{2}{*}{ Iapetus } & & & & 1.00 & 0.78 & 0.62 \\
\hline & & & & $<$ & $<$ & $<$ \\
\hline
\end{tabular}

Table 2. Lifetimes in the transfer of mass among the satellites of Saturn (see text). We have used $V_{\max }$ from Table 1. Three values are for $f_{\mathrm{PB}}=0^{\circ}, 90^{\circ}, 180^{\circ}$ respectively from top to bottom. For every $f_{\mathrm{PB}}$ the number at left has been computed using the geometrical cross-section, that at right for the gravitational cross-section. The number of the colliding fragments is reported in parentheses (the total number of fragments is 1000).

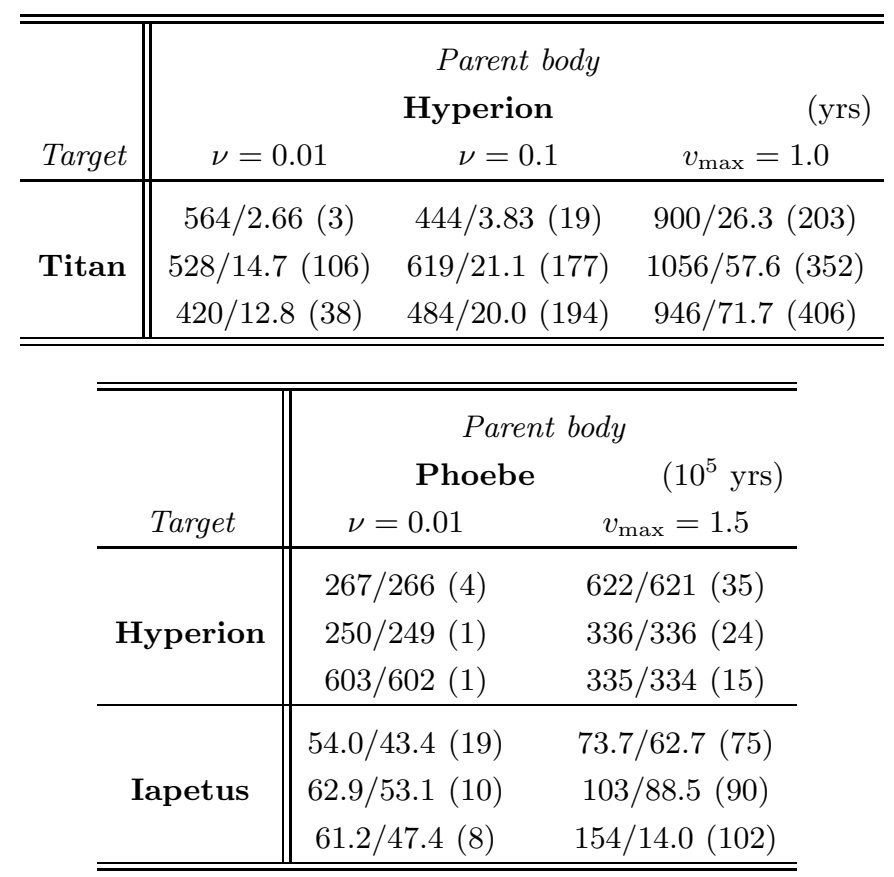


Table 3. Velocity $V_{\max }$ for a percentage $\nu$ of transfer of mass, among the satellites of Uranus (see text, and Table 1).

\begin{tabular}{|c|c|c|c|c|c|c|}
\hline \multirow[b]{2}{*}{ Target } & \multirow[b]{2}{*}{ Puck } & \multirow[b]{2}{*}{ Miranda } & \multicolumn{2}{|c|}{ Parent body } & \multirow[b]{2}{*}{ Titania } & \multirow[b]{2}{*}{ Oberon } \\
\hline & & & Ariel & Umbriel & & \\
\hline \multirow[t]{2}{*}{ Miranda } & 0.89 & & 0.67 & 1.12 & 1.62 & 1.83 \\
\hline & 1.20 & & 1.25 & 2.90 & $<$ & $<$ \\
\hline \multirow[t]{2}{*}{ Ariel } & 1.55 & 0.68 & & 0.48 & 1.03 & 1.27 \\
\hline & 1.92 & 1.00 & & 0.87 & $<$ & $<$ \\
\hline \multirow[t]{2}{*}{ Umbriel } & 2.00 & 1.17 & 0.48 & & 0.58 & 0.84 \\
\hline & 2.35 & 1.55 & 0.67 & & 1.17 & 2.52 \\
\hline \multirow[t]{2}{*}{ Titania } & 2.50 & 1.72 & 1.07 & 0.59 & & 0.28 \\
\hline & 2.76 & 2.05 & 1.32 & 0.79 & & 0.50 \\
\hline \multirow[t]{2}{*}{ Oberon } & 2.72 & 1.97 & 1.34 & 0.87 & 0.29 & \\
\hline & 2.93 & 2.25 & 1.57 & 1.10 & 0.40 & \\
\hline
\end{tabular}

Table 4. Lifetimes in the transfer of mass among the satellites of Uranus (see text, and Table 2). In every box the numbers at the top has been computed supposing a $V_{\max }$ such that $\nu=0.01$. The values at the bottom has been computed for a larger value of $V_{\max }$ : for the cases labelled with "-" $V_{\max }=1 \mathrm{~km} \mathrm{~s}^{-1}$, while for the cases labelled with "+" $V_{\max }=1.5 \mathrm{~km} \mathrm{~s}$.

\begin{tabular}{|c|c|c|c|c|c|c|}
\hline Target & Puck & Miranda & Ariel & $\begin{array}{l}n t \text { body } \\
\text { Umbriel }\end{array}$ & Titania & Oberon \\
\hline Miranda & $\begin{array}{l}19.6 / 16.3(6) \\
33.1 / 29.9(68)^{+}\end{array}$ & & $\begin{array}{l}40.4 / 33.0(10) \\
55.2 / 48.5(66)^{-}\end{array}$ & $\begin{array}{l}76.3 / 71.8(10) \\
90.9 / 86.1(50)^{+}\end{array}$ & & \\
\hline Ariel & & $\begin{array}{l}55.2 / 35.1(6) \\
143 / 116(120)^{+}\end{array}$ & & $\begin{array}{l}19.9 / 10.7(9) \\
91.5 / 73.6(130)^{-}\end{array}$ & $\begin{array}{l}56.9 / 48.3(12) \\
163 / 151(60)^{+}\end{array}$ & \\
\hline Umbriel & & & $\begin{array}{l}34.3 / 14.6(5) \\
170 / 123(101)^{-}\end{array}$ & & $\begin{array}{l}112 / 77.7(10) \\
503 / 443(171)^{+}\end{array}$ & $\begin{array}{l}123 / 94.2(12) \\
689 / 639(98)^{+}\end{array}$ \\
\hline Titania & & & $\begin{array}{l}65.7 / 37.1(2) \\
496 / 360(36)^{+}\end{array}$ & $\begin{array}{l}97.3 / 31.7(4) \\
491 / 244(67)^{-}\end{array}$ & & $\begin{array}{l}92.1 / 15.1(7) \\
860 / 475(230)^{-}\end{array}$ \\
\hline Oberon & & & & $\begin{array}{l}217 / 109(2) \\
1711 / 1100(70)^{+}\end{array}$ & $\begin{array}{l}234 / 32.6(7) \\
1706 / 685(217)^{-}\end{array}$ & \\
\hline
\end{tabular}

\subsection{Saturn's satellites}

Titan is the most interesting target for possible transfer of mass, not only in the system of Saturn, but in the entire Solar System. The importance of Titan, being the largest satellite after Ganymede, with a diameter of $5150 \mathrm{~km}$, is enhanced by the presence of a dense atmosphere, composed mainly of molecular nitrogen and argon and methane, with some amounts of organic compounds and water. In many aspects the atmosphere of Titan is similar to the supposed primordial atmosphere of the Earth. For the transfer of mass, as can be seen in Table 1, Hyperion is the most probable source requiring a $V_{\max }$ only about $0.4 \mathrm{~km} \mathrm{~s}^{-1}$ to have $\nu=0.1$. The lifetimes are very short, being of the order of $10^{3} \mathrm{yrs}$, even in the conservative computation where we neglect the effect of gravitational focusing; taking this into account one may obtain a $\mathcal{T}$ of only some tens of years (due to the large mass of Titan, the effect is very strong). The infall of fragments onto Titan has been also computed with the aid of numerical integrations of the orbits (Farinella et al. 1997; Matteoli 1995, Thesis, unpublished). According to their computations, the time-scale for the exhaustion of most of the potential projectiles has been estimated of the order of $10^{3}$ years (closer to the geometrical cross-section than to the gravitationally enhanced one). Nevertheless, a significant number of collisions takes place in the first decades. This behaviour seems consistent with the qualitative argument presented in Sect. 2. A detailed comparison between the results of their numeric integrations and ours is difficult, as the modeling of fragments' ejection are different, but a general comparison shows fair agreement. 
In Fig. 7 the distribution of the impact velocity of the projectiles colliding with Titan is represented. The lower limit corresponds, obviously, to the Titan escape velocity. The distribution assumes that $f_{\mathrm{PB}}=90^{\circ}$ and $V_{\max }=1 \mathrm{~km} \mathrm{~s}^{-1}$.

If this bombardment was effectively suffered by Titan, its influence on the formation and evolution of the atmosphere certainly has been very important. This problem has been extensively discussed in Farinella et al. (1997). According to their conclusions, the existence of Titan's atmosphere may be connected to the infall of fragments from Hyperion. Taking into account a catastrophic disruption of Hyperion and a transfer fraction of $50 \%$, the estimated mass accreted by Titan is about $(1 \div 3.5) \times 10^{19} \mathrm{~kg}$, while the estimated mass of Titan's atmosphere is of the order of $0.9 \times 10^{19} \mathrm{~kg}$. A quantitative modeling of the formation of the Titan's atmosphere should require taking into account the chemical reactions induced by the impacts. These last aspects have been discussed in Bar-Nun et al. (1970), Chyba \& Sagan (1992), McKay \& Borucki (1997), see these papers, the references therein, and also Marchi (1998, Thesis, unpublished) for a discussion of these points and of other open problems (for example, the observed abundance of $\mathrm{N}_{2}$, which cannot be explained by collisional processes only).

Note also that the previous (quantitative) conclusions are not affected at all by not having considered the possible impacts of the fragments with bodies different from Titan, including Hyperion. In fact Titan by far exceeds the other satellites in size, and thus in cross section (see again Farinella et al. 1990).

We have considered other two cases in the Saturn's system. Some authors have invoked the hypothesis of a transfer of mass in order to explain the observational features of the satellites Iapetus and Hyperion (Buratti 1995; Hamilton 1997). These features have a lower albedo than the other satellites of the system. Phoebe is the possible candidate source. The hypothesis of a strong contribution to the impacts (and then craters) recorded on the surface of Iapetus due to a mass-transfer process, i.e. due to projectiles all coming from similar orbits, should also explain the observed leading-trailing asymmetry between the Iapetus hemispheres (we recall that Iapetus' spin is tidally synchronized with the orbital motion). The results listed in Table 1 show that a transfer of mass from Phoebe to Iapetus (less effectively, to Hyperion), is possible. Note in Table 2 that the transfer time-scale is now $\sim 10^{7} \mathrm{yr}$. We intend to study more extensively the case of Iapetus in a forthcoming paper.

\subsection{Uranus' satellites}

The Uranus' system holds twenty-five pairs of interest when discussing the mass transfer mechanism. Some of the selected objects can be considered both targets and parent bodies. None of these satellites has an atmosphere, since all of them are too small to retain an atmospheric layer.
Nevertheless the results can be used to study the high craterization of the surfaces of the objects (Smith et al. 1986). In this respect it must be taken into account that a particular parent body can supply the fragments for the transfer of mass to different targets simultaneously. Moreover it is worth noting that the efficiency of the transfer does not always remain the same when interchanging target with parent body (see Table 3 for the pairs Titania-Miranda, Titania-Ariel, Oberon-Miranda and Oberon-Ariel, and also Fig. 4). This can be easily understood considering that it is not dynamically equivalent to switch from an internal orbit to an external one or vice versa.

Finally, the mean lifetimes (Table 4) are, as in the case of Titan-Hyperion, of the order of $10^{3} \mathrm{yr}$.

The mass transfer may have given observable effects concerning the cratering record. Two populations have been defined to describe the cratering record in the Uranus system (Strom 1987); Population I craters are present on the surface of Oberon, Umbriel and in the heavily craterized (MHC) region of Miranda, while Population II craters are present on Titania, Ariel and in the Miranda region with a light cratering (MLC). The population II may be explained as a result of collisions with the fragments outcoming from a breakup process (Holsapple \& Schmidt 1982). The required total mass should be around $M_{\text {tot }} \sim 10^{16} \mathrm{~kg}$ for Titania, $\sim 10^{15} \mathrm{~kg}$ for Ariel $e \sim 10^{12} \mathrm{~kg}$ for Miranda. If we assume that those fragments are coming from the closest satellite as a parent body, we obtain mass ratios respectively of $10^{-9}, 10^{-7}$ and $10^{-6}$. Moreover, on the basis of the data concerning the Populations II craters (Strom 1987) and with the same method discussed by Holsapple \& Schmidt (1982), the mass distribution of the possible projectiles is qualitatively consistent with a power law $m^{-q}$ with $q$ between 1.7 and 2. Thus mass transfer processes are effective and reliable candidates as sources of Population II craters.

\section{Appendix}

Equations (9) and (10) can be generalized to eccentric orbits of the parent body. The vertices of the triangle in the plane $(Q, q)$ have to be computed in this case. We have:

$q_{\mathrm{C}}=Q_{\mathrm{C}}=\frac{a_{\mathrm{PB}}\left(1-e_{\mathrm{PB}}^{2}\right)}{1+e_{\mathrm{PB}} \cos f_{\mathrm{PB}}}$.

Equations (4) and (5) are now rewritten as:

$\mathcal{E}_{\mathrm{MIN}}=\mathcal{E}_{0}+\frac{1}{2} V_{\max }^{2}-V_{0} V_{\max }$

$\mathcal{E}_{\mathrm{MAX}}=\mathcal{E}_{0}+\frac{1}{2} V_{\text {max }}^{2}+V_{0} V_{\text {max }}$

where $V_{0}$ is the velocity of the parent body at the moment of the breakup, and $\mathcal{E}_{0}$ its energy. Thus (4)-(5) give

$Q_{\mathrm{A}}=Q_{\mathrm{C}} \quad q_{\mathrm{A}}=\frac{\mu}{\mathcal{E}_{\mathrm{MIN}}}=Q_{\mathrm{c}}$ 

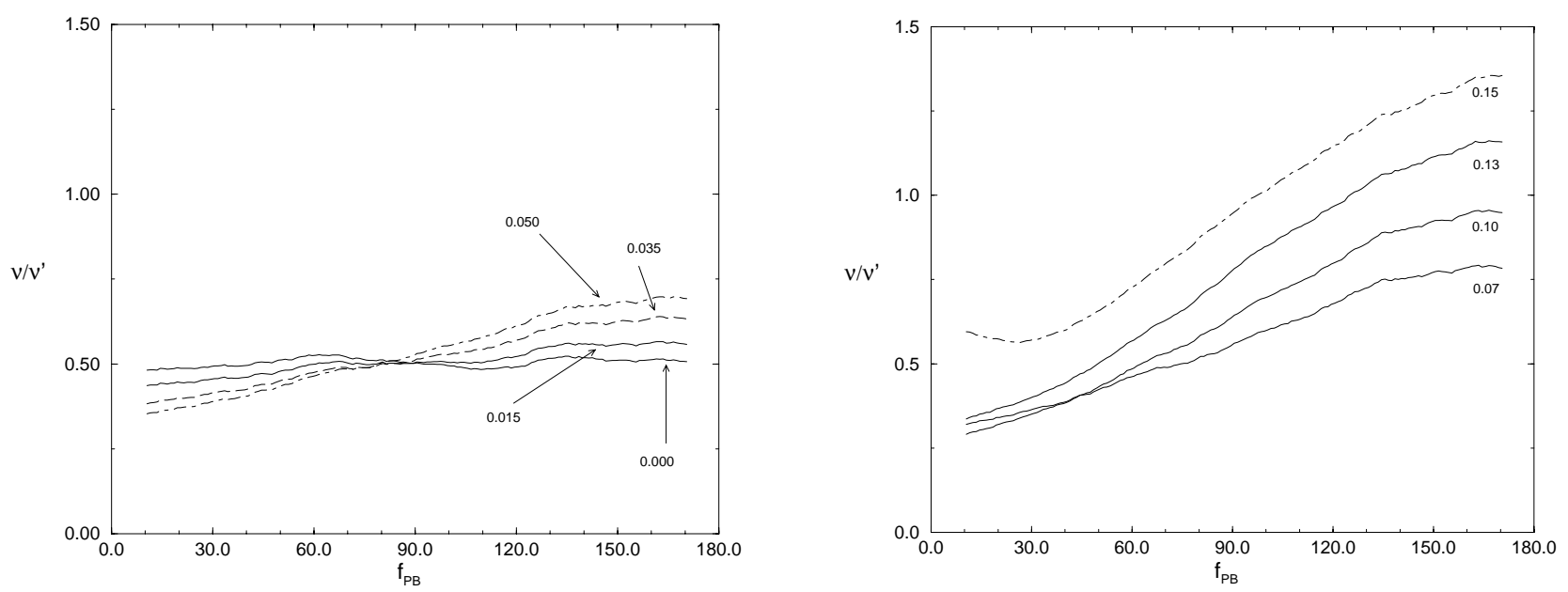

Fig. 8. Mass transfer from Hyperion to Titan: comparison between the exact value of $\nu$ and that computed by the formula $\nu^{\prime}$, as function of the anomaly of the parent body $f_{\mathrm{PB}}$. The number are the eccentricities of the parent body $e_{\mathrm{PB}}$. $V_{\mathrm{max}}$ is equal to $1 \mathrm{~km} \mathrm{~s}^{-1}$.

$Q_{\mathrm{B}}=-\frac{\mu}{\mathcal{E}_{\mathrm{MAX}}}-q_{\mathrm{C}} \quad q_{\mathrm{B}}=q_{\mathrm{C}}$

and the area of the triangle, i.e. of the region IV, is

$$
\begin{aligned}
S_{\mathrm{IV}} & =\frac{1}{2}\left(q_{\mathrm{C}}-q_{\mathrm{A}}\right)\left(Q_{\mathrm{B}}-Q_{\mathrm{C}}\right) \\
& =-\frac{1}{2}\left(2 q_{\mathrm{C}}+\frac{\mu}{\mathcal{E}_{\mathrm{MIN}}}\right)\left(2 q_{\mathrm{C}}+\frac{\mu}{\mathcal{E}_{\mathrm{MAX}}}\right) .
\end{aligned}
$$

The point labelled as $Z$, useful to define the geometric capture region (IV), is now defined by the coordinates $\left(q_{0}, Q_{0}\right)$; nevertheless, the intersection conditions presented in Fig. 3 remain the same. In conclusion, we get:

$\nu^{\prime}=\left(\frac{Q_{0}+\frac{\mu}{\mathcal{E}_{\mathrm{MIN}}}+q_{\mathrm{C}}}{\frac{\mu}{\mathcal{E}_{\mathrm{MIN}}}+2 q_{\mathrm{C}}}\right)^{2}$

for case a, and

$\nu^{\prime}=\left(\frac{q_{0}+\frac{\mu}{\mathcal{E}_{\mathrm{MAX}}}+q_{\mathrm{C}}}{\frac{\mu}{\mathcal{E}_{\mathrm{MAX}}}+2 q_{\mathrm{C}}}\right)^{2}$

for case $c$.

We have tested the formulae comparing the value of $\nu^{\prime}$ with $\nu$ given by a numerical procedure. The most critical parameters are $f_{\mathrm{PB}}$ and $e_{\mathrm{PB}}$, because they strongly affect the distribution of the orbits of the fragments. In Fig. 8 the behavior of the ratio $\nu / \nu^{\prime}$ as a function of $f_{\mathrm{PB}}$ is plotted, in the case Titan-Hyperion, with $V_{\max }=1 \mathrm{~km} \mathrm{~s}^{-1}$. At left the plot shows four cases at low eccentricities $\left(e_{\mathrm{PB}} \leq\right.$ $0.05)$, and at right cases at high eccentricities. It must be taken into account that among the satellites in the Solar System, only four objects have eccentricities higher than 0.05: Himalia (Jupiter, 0.163), Hyperion (Saturn, 0.1042), Phoebe (Saturn, 0.163) and Nereid (Neptune, 0.7512). The analysis shows that $\nu \simeq \nu^{\prime} / 2$ for low eccentricities, while for high eccentricity it is necessary to introduce a corrective factor $\gamma\left(f_{\mathrm{PB}}, e_{\mathrm{PB}}\right)$, so that $\nu \simeq \gamma\left(f_{\mathrm{PB}}, e_{\mathrm{PB}}\right) \nu^{\prime}$, to be estimated individually for the particular object under consideration.
The discrepancy between $\nu$ and $\nu^{\prime}$ is due mainly to the non uniformity of the distribution of the objects in region III (so that the ratio between the areas is different from $\nu$ ) and also to the assumption of exact triangular shape for the same region.

Acknowledgements. We discussed the first ideas of this work with Paolo Farinella, who is recently deceased. We thank Francesco Marzari (Padua University) for remarks and suggestions. We are also grateful to an anonymous referee for useful criticisms.

This work as been funded by MURST-Cofin98, through the Padua and Pisa groups, lead by C. Barbieri and P. Paolicchi.

\section{References}

Bar-Nun, A., Bar-Nun, N., Bauer, S. H., \& Sagan, S. 1970, Science, 168, 470

Bertotti, B., \& Farinella, P. 1990 (Kluwer Academic Publishers)

Bottke, W. F., Nolan, M. C., Greenberg, R., \& Kolvoord, R. A. 1994, Icarus, 107, 255

Brown, H. 1952, The atmosphere of the Earth and Planets, 2nd ed., ed. Kuiper, 258

Buratti, B. J. 1995, Icarus, 115, 219

Chyba, C. F., \& Sagan C. 1992. Nature, 355, 125

Dell'Oro, A., Marzari, F., Paolicchi, P., \& Vanzani, V. 2001, A\&A, 366, 1053

Dell'Oro, A., \& Paolicchi, P. 1998, Icarus, 136, in preparation, 328

Farinella, P. 1986, Proc. NATO Conf. on Saturn

Farinella, P., Marzari, F., \& Matteoli, S. 1997, AJ, 113, 2312

Farinella, P., Paolicchi, P., Strom, R. G., Kargel, J. S., \& Zappalà, V. 1990, Icarus, 83, 186

Gladman, B. J., Burns, J. A., Duncan, M. J., \& Levison, H. F. 1995, Icarus, 118, 302

Greenberg, R. 1982, AJ, 87, 185

Hamilton, D. P. 1997, Am. Astron. Soc., DPS Meeting No. 29, Abstract, No. 20.02

Holsapple, K. A., \& Schmidt, R. M. 1982, J. Geophys. Res., 87,1849

Kresák, L. 1967, B.A.C., 18(1), 27 
McKay, C. P., \& Borucki, W. J. 1997, Science, 276, 390

Melosh, H. J., \& Vickery, A. M. 1989, Nature, 338, 487

Melosh, H. J. Ryan, E. V. Asphaug, E. J. Geophys. Res., 97(E9), 14735

Ozima, M., \& Igarashi, G. 1989, in Origin and evolution of planetary and satellite atmospheres (The University of Arizona press, Tucson), 306

Paolicchi, P., Cellino, A., Farinella, P., \& Zappala, V. 1989, Icarus, 77,187

Paolicchi, P., Verlicchi, A., \& Cellino, A. 1996, Icarus, 121, 126

Ryan, E. V., \& Melosh, H. J. 1998, Icarus, 133, 1

Safronov, V. S. 1972, NASA TT F 677

Safronov, V. S. 1979, in Asteroids (Tucson, Ariz., University of Arizona Press), 975

Smith, B. A., Soderblom, L., Beebe, R. F., et al. 1981, Science, 212,163
Smith, B. A., Soderblom, L., Batson, R., et al. 1982, Science, 215,505

Smith, B. A., Soderblom, L. A., Beebe, R., et al. 1986, Science, 233, 43

Strom, R. G. 1987, Icarus, 70, 517

Tanga, P., Cellino, A., Michel, P., et al. 1999, Icarus, 141, 65

Vedder, J. D. 1996, Icarus, 123, 436

Vedder, J. D. 1998, Icarus, 131, 283

Walker, J. C. G. 1986, Nature, 68, 87

Wetherill, G. W. 1967, J. Geophys. Res., 72, 2429

Zahnle, K., Levison, H., Dones, L., \& Schenk, P. 1999, Lunar and Planetary Science XXX, Abstract No. 1776

Zahnle, K., Dones, L., \& Schenk, P. 2000, Lunar and Planetary Science XXXI, Abstract No. 2053

Zappalà, V., Cellino, A., Dell'Oro, A., Migliorini, F., \& Paolicchi, P. 1996, Icarus, 124, 156 